THIS PAPER MUST BE CITED AS:

Advanced Materials, Vol 19(20), 2007, 3364-3367

\title{
Cathodic and Anodic Material Diffusion in Polymer/Semiconductor- Nanocrystal Composite Devices
}

\author{
By Diego E. Gallardo*, Cristina Bertoni, Steve Dunn, Nikolai Gaponik, Alexander Eychmüller \\ Dr D.E. Gallardo, Dr C. Bertoni, Dr S. Dunn. Microsystems and Nanotechnology Centre. Building 30. Cranfield University. \\ MK43 0AL Bedford. England. E-mail: d.e.gallardo@cranfield.ac.uk
}

Dr N. Gaponik, Prof A. Eychmüller. Physical Chemistry/Electrochemistry. TU Dresden. Bergstr. $66 \mathrm{~b}, 01062$ Dresden. Germany

In the present day, the information technologies and telecommunications sector continually increase their demand for low cost, low power consumption, high performance electroluminescent devices for display applications. Furthermore, general lighting applications, such as white light and large array colour displays, would also benefit from an increase in the overall efficiency. Several technologies are being investigated to fulfill these needs, such as organic light emitting diodes (OLED), polymeric light emitting diodes (PLED) and field effect emission devices. A new and promising technology is light emitting devices (LEDs) based on nanostructured materials. With organic LEDs (OLEDs) already making an impact on the market in an increasingly large number of applications, hybrid technologies based on organic/inorganic nano-composites are a potential the next step. The incorporation of high-efficiency fluorescent semiconductor nanoparticles has been shown to have a beneficial effect on device performance,[1] modify the colour output from the device[2] and provide a simplified route to generation of LED type devices.[3]

Fluorescent semiconductor crystallites offer a high spectral purity and excellent luminescence quantum efficiency. These properties stem from the confinement of the carriers within the nanocrystals.[4] However, this confinement also reduces the carrier transport capabilities of the nanocrystals.[5,6] Carrier transport is reduced further when the nanocrystals are embedded in a polymer matrix.[3] Therefore, most devices incorporating semi-conductor nanocrystals work at fields in the order of $107 \mathrm{~V} / \mathrm{m}$ or higher. The implications of high applied fields in device degradation have been investigated by various groups in other structures where similar values of field are applied, particularly in organic LEDs (OLEDs). Gao et al.[7] have described damage in hybrid polymer/nanocrystal devices caused by the current-induced oxidation of the aluminum cathode. In the case of OLEDs, the formation of dark spots has been related to the localized degradation of metallic cathodes, caused by electromigration at high operational fields.[8,9] Furthermore, Gautier et al.[10] found that fieldinduced damage on ITO electrodes can lead to device failure.

Here, electrode diffusion-related degradation mechanisms in nanoparticles/polymer composites LEDs have been studied. The structure used for this purpose has been described in detail elsewhere.[3,11] Briefly, a composite multilayer made of a dielectric polymer (poly-diallyl-dimethyl-ammonium, PDDA) and CdTe nanoparticles was sandwiched between an aluminum cathode and an ITO anode. The multilayer was deposited through a layer-by-layer process carried out in air. The thicknesses of the different materials are $35 \mathrm{~nm}, 220 \mathrm{~nm}$ and $130 \mathrm{~nm}$ for aluminum, multilayer and ITO, respectively. Each substrate had up to 6 identical independently addressable devices with an active area of $4 \mathrm{~mm} 2$ (Fig. 1). Four such devices were biased at $4.0 \mathrm{~V}$ for $15 \mathrm{~s}$, two under moderate vacuum (10-5 mbar), and another two in air.

The electric field was approximately $1.8 \times 107 \mathrm{~V} / \mathrm{m}$ and measured currents were in all cases below $1 \mu \mathrm{A}(25 \mu \mathrm{A} / \mathrm{cm} 2)$, and dropping $80-100 \%$ over the measurement period. Compositional depth profiles were acquired by secondary ion mass 
spectrometry (SIMS). The species examined were A127+, In115+, and O16- The composition profiles were taken in both active and passive areas. Active areas were those in which cathode and anode cross, and across which the field is applied, noted as A1 in Fig. 1. Passive areas were those areas which did not receive any applied field. Two different types of passive areas were studied: aluminum plus multilayer, noted as P1 in Fig. 1, and multilayer plus ITO, noted as P2 in Fig 1. Investigation of the passive areas $\mathrm{P} 1$ and $\mathrm{P} 2$ gives information equivalent to a non-biased active area A1. However, there is an advantage in that the aluminum and ITO distributions that can be studied independently.

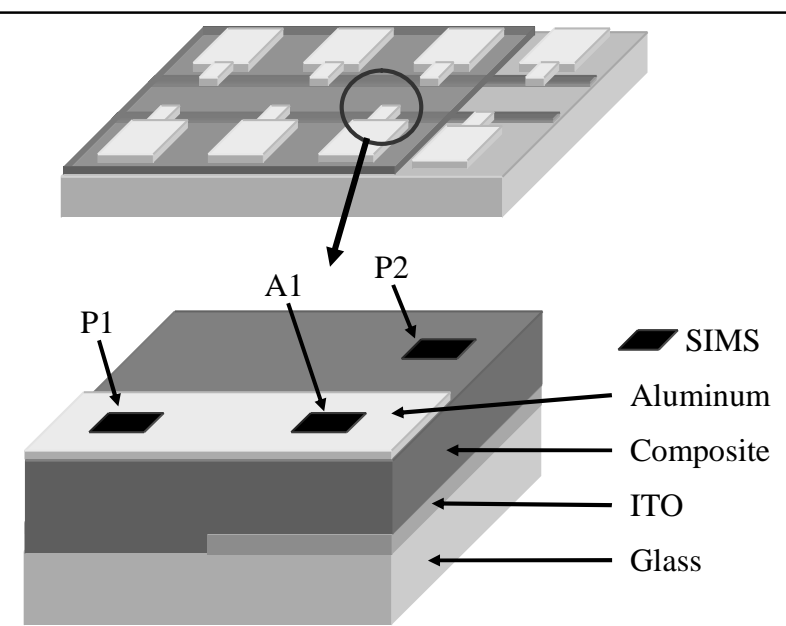

Figure 1. The device structure consists in a multilayer of PDDA/CdTe sandwiched between an aluminum anode and an ITO cathode. Device currents were $25 \mu \mathrm{A} / \mathrm{cm} 2$ (maximum), with an applied bias of $4 \mathrm{~V}$ (or 107 $\mathrm{V} / \mathrm{m}$ ). The areas analyzed with SIMS are marked with black squares. The device's active area is that where cathode and anode cross (A1). Two different types of passive areas are studied: aluminum plus multilayer (P1), and multilayer plus ITO (P2).

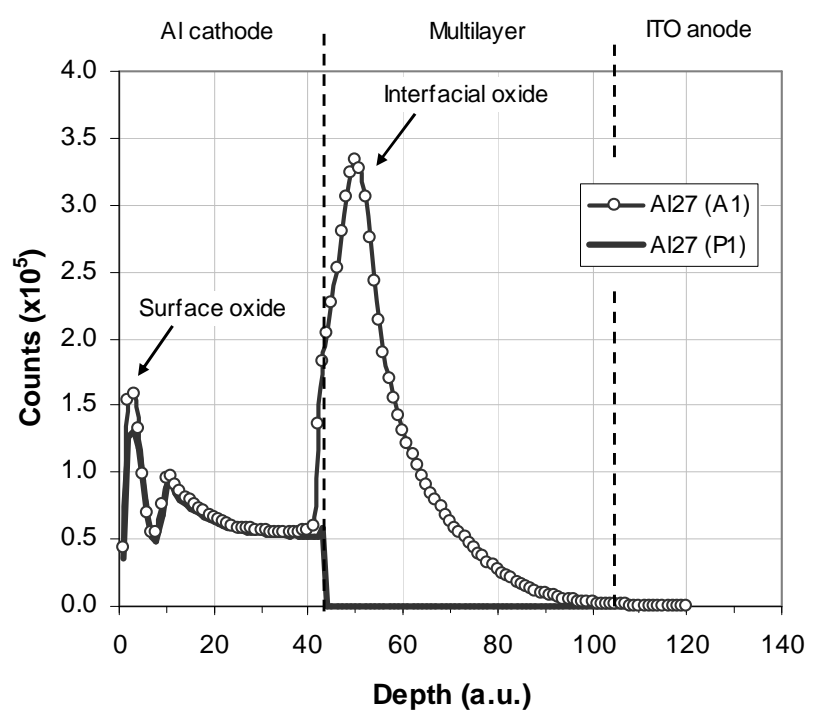

Figure 2. Aluminum depth profiles from areas A1 (circles) and P1 (line). The discontinuous vertical lines mark the interfaces between the electrodes and the multilayer. The location of these interfaces is an indication only, derived from AFM and WLI measurements. Although the aluminum has been negatively biased, it can be seen that there is a significant diffusion of aluminum into the composite in area A1. This diffusion is caused by the strong applied field. Electromigration is ruled out due to the low current densities measured during the biasing of the devices. 
Depth calibration was done by measuring the depth of the SIMS trenches by AFM (contact mode) and white light interferometry. It is important to note that due to the chemical complexity of the structure, no milling rates could be calculated accurately.[12] Consequently, depth information in figures 2 to 5 is only approximate and serves only to give an idea of the relative position of the different layers. There was no substantial difference between devices biased in air and in moderate vacuum. All the devices presented qualitatively the same features and properties. Therefore, for simplicity of description, the results presented here will correspond to the A1, P1 and P2 areas from a single device, but the discussion is appropriate to the other three devices on the substrate.

The aluminum depth profiles for both A1 and P1 areas are shown in fig. 2. The vertical discontinuous lines are added to indicate the position of the organic/inorganic composite. Both A1 and P1 areas showed an aluminum peak near the top surface of the sample. This peak is associated with the passivation oxide film that forms on aluminum when exposed to the air. After this feature the intensity stabilized, with little variation between the readings from active and passive areas. It is reasonable to link this region to the "bulk" of the cathode film. After a small peak at the interface between the cathode and the organic multilayer, the aluminum intensity falls sharply in the passive area, and remained practically zero for the rest of the analysis. The small peak can be related to a thin interfacial alumina layer formed during the deposition of the cathode. However, in the active area the aluminum intensity not only did not fall at the interface with the multilayer, but it presented a wide peak with a tail extending across all the composite film. This tail is attributed to the diffusion of aluminum atoms during the biasing of the device. The possible influence of the formation of pinholes during cathode deposition on the aluminum distribution within the organic layer was discarded due to the sharp aluminum edge in the depth profile shown by the adjacent P1 area (see Fig. 1). At this point it is useful to remember that the variation in composition with depth into the sample results in different milling rates. This is known as matrix effect.[12] Thus, the interfacial peak in the active areas after the cathode/multilayer interface must not be interpreted in terms of an aluminum abundance higher than in the bulk of the electrode, but as a change in the chemical nature of the materials present at that depth.[13]

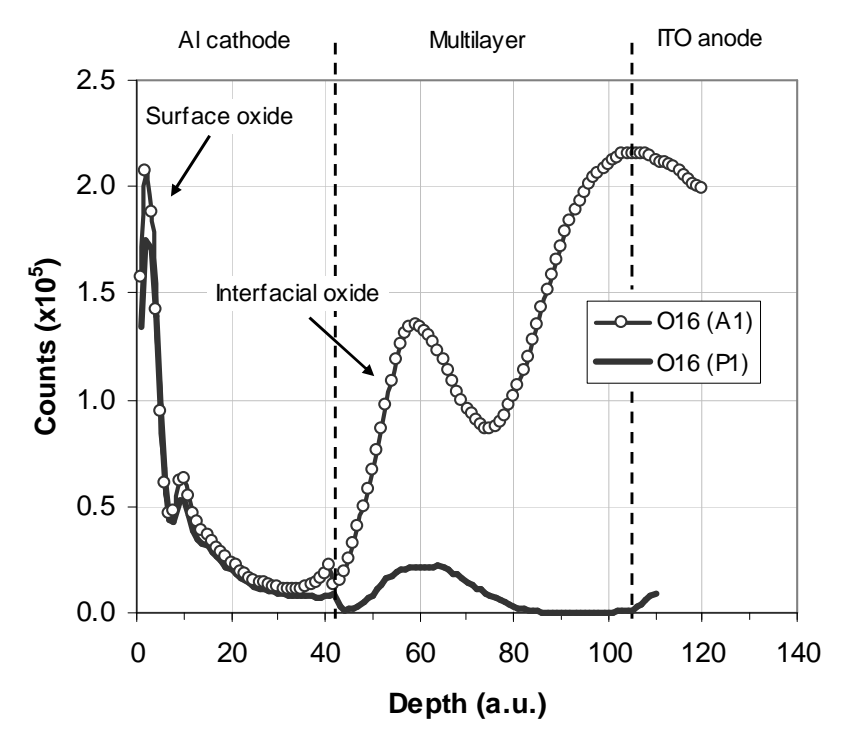

Figure 3. Oxygen depth profiles from the same A1 and P1 areas as in figure 2. The peak beginning at the cathode/multilayer interface is substantially higher in the active area, denoting an extra content of oxygen at this region. This extra oxygen is associated with the oxidation of the diffused aluminum. The oxygen peak in A1 near the interface with the anode is due to the diffusion of oxygen from the ITO (connected with the In as shown further below).

Figure 3 shows the oxygen profiles of the same A1 and P1 areas of Figure 2. Both profiles showed a first peak at the surface in contact with air, indicating the presence of surface oxide, as described before for aluminum. Both intensities then decayed smoothly with the depth into the cathode, showing similar intensities for both active and passive areas. As with aluminum, the differences between active and passive areas start at the cathode/multilayer interface. The active area presented a clear second peak in oxygen just after the interface; that peak was also present in the passive area P1, but its associated readings were around $85 \%$ lower than in the active area. The agreement in the data collected from the different devices indicates a homogeneous composition for active and passive areas before any of the devices were biased. Therefore, the second peak in A1 had evolved while the device was biased. The evolution of this peak is associated with a change in chemical environment for the aluminum, indicating the presence of incorporated oxygen. The extra oxygen in area A1 is related to the oxidation of the diffused aluminum, which was absorbed from the environment surrounding the device (at 10- 
5 mbar there would be enough oxygen present to oxidize the aluminum). This diffusion of environmental oxygen into polymeric layers during device operations has been already reported in other structures.[7,8] The active area also presented a third oxygen peak deeper into the organic layer that is absent from the profile of the P1 area, but it is present in the P2 area (Fig. 5). This is associated with the ITO anode (Figures. 4 and 5).

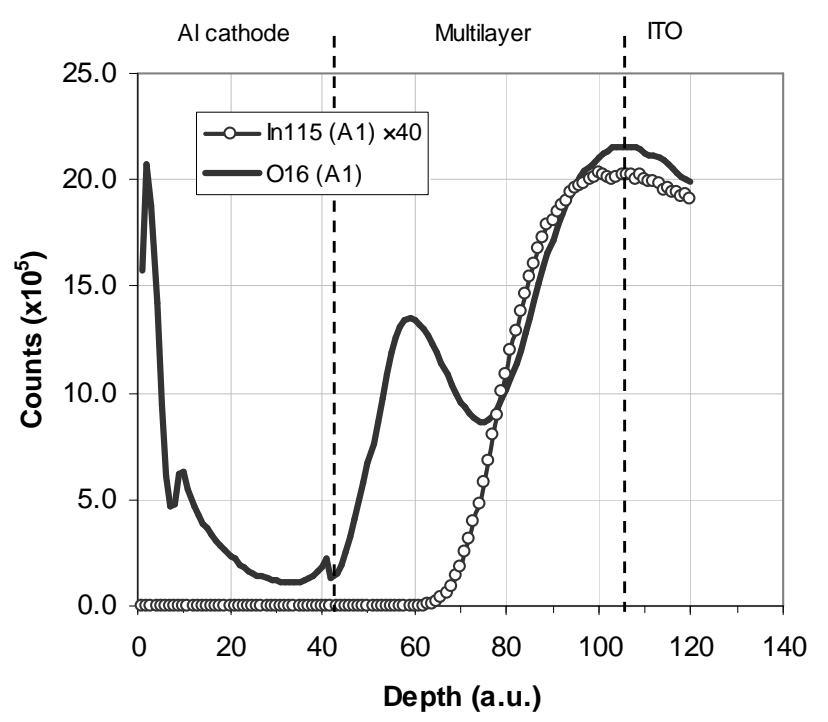

Figure 4. Indium and oxygen depth profiles from area A1. The indium has diffused for more than $65 \%$ of the multilayer thickness. The oxygen peak near the anode matches the indium profile at that depth, indicating that both elements are associated.

The indium's depth profile corresponding to the active area is shown in figure 4 together with, for reference, that of oxygen. It can be seen that substantial indium contamination is found in the organic multilayer. The indium penetration depth (corresponding to an indium count lower than $1 \%$ of the peak value) is approximately equivalent to $65 \%$ of the multilayer thickness. The shape of the indium profile matches the third peak of oxygen, suggesting that this oxygen is associated with the diffused indium in the form of indium oxide. Figure 5 shows the indium and oxygen profiles for a P2 area. Indium diffusion into the organic multilayer can also be observed in this area, with a penetration of $40 \%$ in thickness into the multilayer. Indium diffusion is therefore present even in areas in which a field has not been applied. The indium penetration is carried further into the composite by an additional $25 \%$ with the application of an electric field.

The indium diffusion found in the present work has been discussed already in the OLED-related literature. Hirose et al. $[14,15]$ reported the spontaneous diffusion of reactive metals like indium and aluminum deposited on top of an organic layer. Schlatmann et al.[16] studied the diffusion of underlying indium into organic layers in OLEDs prior to device operation, whereas Gautier et al.[10] reported the diffusion of indium during operation. Chao et al.[17] explained the field induced diffusion as a consequence of the decomposition of the ITO.

The case of the aluminum diffusion towards the anode requires a different explanation. The measured currents were too low to produce substantial electromigration[18] or thermal diffusion. Studies of ionic drift of metal into organic layers have demonstrated that aluminum can penetrate deep into organic layers.[15,19-21] However, these studies are based in metal gates biased positively, whereas in this work the aluminum is negatively biased and diffuses towards the anode. Also, the diffusion of metallic aluminum across the multilayer should induce an increase in current due to the enhanced field. In the present study, the current did not increase but fell substantially. Consequently, it seems that the diffused aluminum is already oxidized or oxidizes very quickly. The mechanism proposed here is based on the explanation given by Mott[22] for the growth of the passivation layer in aluminum. Mott explained that an electron transfer to adsorbed oxygen atoms creates an electric field that forces an ionic current across the thin oxide layer. These ions then get oxidized and thicken the oxide layer, until a point in which electrons cannot tunnel over the thickness of the oxide. In the presence of a strong applied electric field, as is the case of the samples presented here, electrons can tunnel over thicker layers of oxide, allowing a further growth of the oxide. The growth of a thicker aluminum oxide at the cathode/multilayer interface explains the loss of conductivity noticed in the device. The gradual decay of aluminum intensity within the composite suggests that the diffused oxide consists of dendritic filaments, rather than maintaining a flat interface. The dendrites might originate from locally field-enhanced areas and penetrate the organic multilayer at weak structural points. These are likely to be located within the polymer, as the nanoparticles are comparatively tougher due to their solid crystalline structure. 
It is important to remark that, despite of the presence of the nanoparticles within the polymer, no specific role is played by them in relation with the diffusion process. Their only influence might be reduced to an effect in the SIMS milling rates through the composite. Thus, the results shown here might be applicable to other structures built with polymers and subjected to high operational fields, like thin-film OLEDs and other opto-electronic devices.

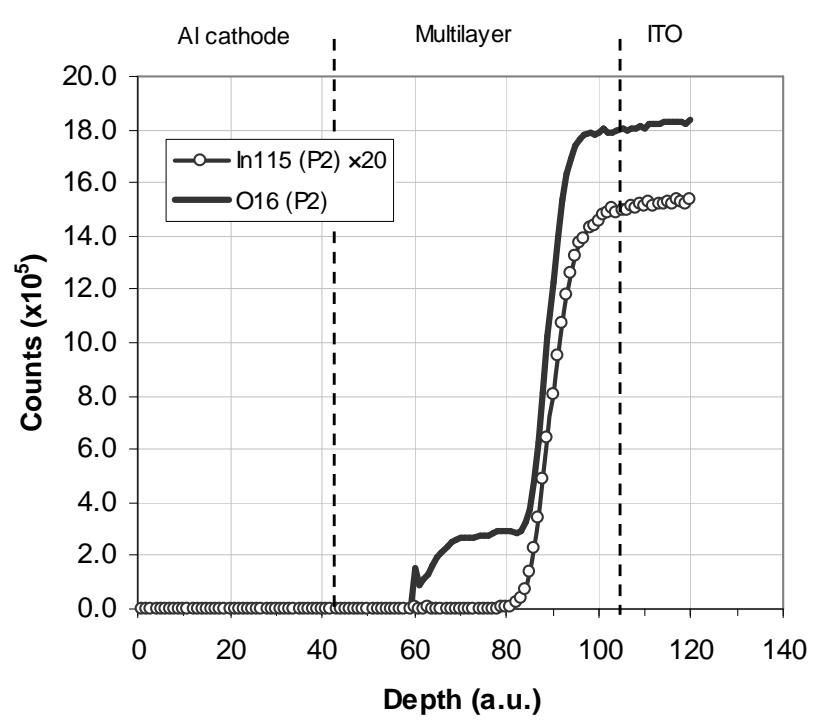

Figure 5. Indium and oxygen depth profiles from area P2. Indium diffusion is present even in areas to which no field has been applied. The indium penetration depth is around $40 \%$ of the multilayer thickness, some $25 \%$ less than in active areas.

Therefore, it is concluded that diffusion of metallic ions from the electrodes is a mechanism involved in the degradation of devices built with organic layers or composites. The diffusion of indium had already been identified as a cause of defects that lead to device degradation.[16,17] A cathode diffusion mechanism due exclusively to the applied field has been found, which can induce structural defects even at low-current regimes. Field-driven aluminum diffusion and oxidation has been shown to produce an insulating barrier that reduces the conductivity of the device, causing irreversible device failure. Suitable cathodic diffusion barriers compatible with electron injection might result in an improvement of device lifetime and performance, both in OLED and hybrid technologies.

\section{Experimental}

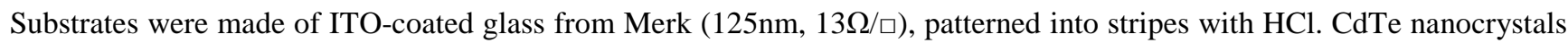
in aqueous solution $(1 \mathrm{mg} / \mathrm{ml})$ were deposited through a layer-by-layer technique. The PDDA water solution concentration was $10 \mathrm{mM}, 0.2 \mathrm{M}$ ionic. The final multilayer presented a CdTe volume fraction of $25-30 \%$. Aluminum cathodes were evaporated on top of the multilayer at $\mathrm{P}<4 \times 10-6 \mathrm{mbar}$, with resultant resistivity between $1.58 \times 10-4 \Omega-\mathrm{cm}$ and $1.19 \times 10-5 \Omega$ $\mathrm{cm}$.

Depth profiles were acquired with a quadrupole-based SIMS, 6500 from Atomika, equipped with a nitrogen gun. The primary ion beam (floating ion gun FLIG5 from Ionoptika) was set at $1.25 \mathrm{kV}$ (N2), with normal incidence on the sample, and a current of 120nA. Depth calibration was done with a Veeco Dimension 3000 AFM in contact mode and a Talysurf CCI 6000 white light interferometer from Taylor and Hobson.

\section{Acknowledgements:}

We would like to thank Mr Richard J. Chater, from Imperial College in London, for his assistance with SIMS analyses.

\section{References}


[1] S. Coe, W.K. Woo, M. Bawendi and V. Bulovic, Nature, 2002, 420, 800-803.

[2] Personal communication, Prof M.C. Petty, Durham University.

[3] C. Bertoni, D. Gallardo, S. Dunn, N. Gaponik and A. Eychmüller, Appl. Phys. Lett., 2007, 90, 034107.

[4] S.V. Gaponenko (1998), in Optical properties of semiconductor nanocrystals, (Eds: S.V. Gaponenko), Cambridge University Press, Cambridge, UK, 1998, 27-54.

[5] C.A. Leatherdale, C.R. Kagan, N.Y. Morgan, S.A. Empedocles, M.A. Kastner and M.G. Bawendi, Phys. Rev. B, 2000, $62,2669-2680$.

[6] N.Y. Morgan, C.A. Leatherdale, M. Drndic, M.V. Jarosz, M.A. Kastner and M. Bawendi, Phys. Rev. B, 2002, 66, 075339-1-9.

[7] M. Gao, B. Richter, S. Kirstein and H. Mohwald, J. Phys. Chem., 1998, 102, 4096-4103.

[8] B.H. Cumpston, I.D. Parker and K.F. Jensen, J. Appl. Phys., 1997, 81, 3716-3720.

[9] B.H. Cumpston and K.F. Jensen, Appl. Phys. Lett., 1996, 69, 3941-3943.

[10] E. Gautier, A. Lorin, J.M. Nunzi, A. Schalchli, J.J. Benattar and D. Vital, Appl. Phys. Lett., 1996, 69, 1071-1073.

[11] D.E. Gallardo, C. Bertoni and S.C. Dunn, Proc. of SPIE, 2005, 5840, 516-522.

[12] A.W. Czanderna (1991), in Ion spectroscopies for surface analysis, (Eds: A.W. Czanderna and D.M. Hercules), Plenum Press, New York, 1991, 25.

[13] E. Cuynen, P. Van Espen, G. Goeminne and H. Terryn, J. Anal. At. Spectrom., 1999, 14, 483-486.

[14] Y. Hirose, A. Kahn, V. Aristov, P. Soukiassian, V. Bulovic and S.R. Forrest, Phys. Rev. B, 1996, 54, $13748-13758$.

[15] Y. Hirose, A. Kahn, V. Aristov and P. Soukiassian, Appl. Phys. Lett., 1996, 68, 217-219.

[16] A.R. Schlatmann, D.W. Floet, A. Hilberer, F. Garten, P.J.M. Smuldars, T.M. Klapwijk and G. Hadziioannou, Appl. Phys. Lett., 1996, 69, 1764-1766.

[17] C.I. Chao, K.R. Chuang and S.A. Chen, Appl. Phys. Lett., 1996, 69, 2894-2896.

[18] I.A. Blech and E. Kinsbron, Thin Solid Films, 1975, 25, 327-334.

[19] A.L.S. Loke, J.T. Wetzel, P.H. Townsend, T. Tanabe, R.N. Vrtis, M.P. Zussman, D. Kumar, C. Ryu and S.S. Wong, IEEE T Electron Dev., 1999, 46, 2178-2187.

[20] A. Mallikarjunan, S.P. Murarka and T.-M. Lu, Appl. Phys. Lett., 2001, 79, 1855-1857.

[21] K.-L. Fang and B.-Y. Tsui, J. Appl. Phys., 2003, 93, 5546-5550.

[22] N.F. Mott, Trans. Faraday Soc., 1947, 429-434. 